\title{
Educación a distancia y mundos virtuales
}

Distance education and virtual worlds

Jaime García Sánchez ${ }^{1}$

Patricia Jáuregui Arias ${ }^{2}$

García S, Jaime. Jáuregui A, Patricia miradas $\mathrm{N}^{\circ} 2$ - 2019 ISSN digital N²539-3812 Págs 163 - 177

Recepción: Enero 31 de 2019

Aprobación: Junio 8 de 2019

Publicación: Junio 30 de 2019

\section{Resumen:}

La educación a distancia ha recorrido un camino largo desde su primera aparición hasta nuestros días. No siempre bien reconocida y casi siempre mal evaluada desde la perspectiva de la educación tradicional presencial, la educación a distancia en su fase virtual ha logrado tener un espacio propio de desarrollo y aceptación. La adopción y utilización creativa de los avances en los distintos medios de comunicación para fines educativos, y de transmisión y recepción de información, ha puesto a esta modalidad educativa en un punto de vanguardia respecto a los modelos tradicionales. El uso de la red de Internet y los adelantos que en ella y de ella se desprenden hacen ver que la educación a distancia tendrá un papel cada vez más relevante en la sociedad global, revolucionando los esquemas del aprendizaje humano mediante su operatividad en mundos interactivos y tridimensionales (realidad virtual y aumentada), que generarán una nueva manera de abordar el aprendizaje humano y de entender la escuela.

Palabras clave: Educación a distancia, Educación virtual, Realidad virtual, Realidad aumentada, Nanotecnologías

\footnotetext{
1 Doctor en educación, docente investigador en el Centro Interdisciplinario de Investigación y Docencia en Educación Técnica (CIIDET), Querétaro, México. egruen59@hotmail.com

2 Doctora en educación, supervisora escolar en la Secretaría de Educación de Guanajuato (SEG), México. ariaspaty@yahoo.com
} 


\section{Abstract}

Distance education has come a long way since its first appearance until today. Not always well recognized and often poorly evaluated from the perspective of traditional classroom education, distance education in their virtual phase has managed to have its own space development and acceptance. The adoption and creative use of advances in the various media to educate and transmit and receive information, has made this educational model a vanguard point over traditional models. The use of Internet and advances in it and they see it emerge that distance education will have an increasingly important role in global society, revolutionizing the patterns of human learning through interactive, operability and dimensional worlds (virtual reality), which will generate a new approach to the human learning and understanding the school.

Keywords: Distance education, Virtual education, Virtual reality, Augmented reality, Nanotechnologies

\section{Una breve visión del origen de la educación a distancia}

La educación a distancia ha transitado, desde sus orígenes, entre el rechazo y la aceptación forzada. La escuela es, en general, quizá, una de las instituciones sociales más reacias a aceptar cambios y flexibilizar tanto sus procesos como sus estructuras. Enmarcada en una relación de poder, la academia tradicional busca el control y el orden burocrático. En ella se desarrollan rituales preestablecidos, producto de una cultura de autoridad en donde el conocimiento que se imparte tiene el fin último del estatus y la imagen pública de quien obtiene los títulos o grados que expide. Una característica distintiva de la misma es su poca flexibilidad para adaptarse rápidamente a los cambios del entorno. Por contraste la educación a distancia desde sus orígenes, ha estado justamente en el lado opuesto de la moneda, beneficiaria y rápidamente asimiladora de todos los cambios que se presentan en rededor, solamente ha sobrevivido gracias a su plasticidad (García, 2005a).

Originalmente dedicada a satisfacer las necesidades de aquellas franjas de la población no susceptibles, económicamente de ser escolarizadas, se inicia tímidamente como una opción para la adquisición de conocimiento y habilidades desde una perspectiva por antonomasia libertaria, en donde la única relación de poder se da entre la forma y los contenidos de los materiales educativos y por la posibilidad de una entrega más o menos oportuna de los mismos. Todo lo demás dependía exclusivamente del aprendiz, de sus motivaciones y de sus capacidades.

Podemos decir que la educación a distancia se inicia propiamente con la invención de la imprenta (Torres, 2001). La posibilidad que proporcionó este instrumento para plasmar en un medio físico las ideas de otros, permitió, para aquellos que estaban preparados para la auto instrucción, romper la relación que hasta ese momento había sido obligada para quienes deseaban aprender algo nuevo; el vínculo entre el maestro y el alumno en un lugar predeterminado: la escuela. 
La primera etapa de la educación a distancia se distinguió por la utilización primordial de materiales impresos vía correo postal y la posibilidad de tener, por el mismo camino, una serie de asesorías para el estudiante, (Educación a distancia [EDUCADIS], 2002). Las limitantes de esta forma de educación, evidentemente, residían en las pocas posibilidades de interacción siendo en muchos sentidos bastante unidireccional, además de estar sujeta a los avatares del tiempo y las distancias para el envío y la recepción del correo. Los cursos por correspondencia, todos ellos para el aprendizaje de habilidades laborales, se dirigían a los sectores económicamente más bajos, con un carácter informal más cercano a los oficios que a una formación profesional con cierto prestigio en la sociedad.

Alejada la mayoría de las veces de los prestigiados centros educativos de la época, la educación a distancia sirvió, -como ha sido hasta la fecha-, para la educación o capacitación de grandes núcleos poblacionales y que, por las propias limitantes tanto geográficas, económicas e ideológicas, los centros educativos tradicionales no podían ser de gran ayuda.

El desarrollo de la radio, la telefonía y la televisión amplió los canales de comunicación social y fueron, para la educación a distancia, la oportunidad de acceder a diferentes infraestructuras comunicacionales para su desarrollo. Bajo la egida de tales medios, se proyectaron otros esquemas tales como las videocintas, las grabadoras y las reproductoras de audio y los proyectores de diapositivas u objetos opacos. Tales medios y artefactos establecieron las bases para que la educación a distancia saltara a una segunda etapa de su desarrollo pues redujeron las distancias y permitieron el desarrollo de nuevas formas de proceder de la didáctica de una manera más creativa.

En esta segunda etapa el trabajo se centró en el diseño de paquetes instruccionales en los cuales se incluían textos planeados para el auto estudio, así como pistas de audio y de video que vinieron a ampliar las potencialidades de este modelo de educación y trajo consigo algunas ventajas como las siguientes: a) La apropiación del uso didáctico de los medios de comunicación, b) La planeación y el diseño previo de los materiales instruccionales y c) El desarrollo de técnicas de auto estudio.

Durante esta etapa, la educación a distancia había permeado ya en la sociedad, su utilización para la educación básica, sobre todo para la alfabetización de adultos y ciertos niveles intermedios por lo que ya es aceptada por las instituciones tradicionales, -no sin cierto desdén-, puesto que representaba en sí misma una contradicción a su orden y métodos preestablecidos.

El desarrollo de la tecnología televisiva y los medios satelitales le permitieron consolidarse como un modelo de educación con amplias y variadas aplicaciones. Podemos sostener que fue la televisión quien le abrió la puerta del reconocimiento formal. A partir de este momento, la educación a distancia abandonaría los sótanos y la imagen de informalidad a que, en cierto sentido, se 
le había otorgado tanto en los ámbitos de la educación tradicional como en el orden social en general. Pese a lo anterior, aún adolecía de interactividad. A pesar de la mayor rapidez en la comunicación, los programas seguían siendo bastante unidireccionales y carecían de la retroalimentación necesaria para que se diera un diálogo educativo más fluido.

La utilización de los satélites permitió a la educación a distancia dar el salto cualitativo a su tercera fase de desarrollo. Ello facilitó el camino para el progreso de la teleconferencia, misma que dio pie a la comunicación en directo y en tiempo real entre los actores del hecho educativo. Mediante tal vía, se pudo generar la retroalimentación necesaria para romper la unidreccionalidad que, hasta ese momento, en mayor o menor medida, había venido caracterizando a este modelo educativo. Permitió, además, establecer una comunicación entre diferentes videoconferenciantes $\mathrm{y}$ sus audiencias, no importando los ámbitos geográficos o las disciplinas, con lo que cualitativamente la calidad de los programas ofertados en esta modalidad creció y las distancias se opacaron ostensiblemente. De este modo, liberada ya de ciertas ataduras tecnológicas y estigmas ideológicos, la educación a distancia inició una carrera explosiva que le ayudó a posicionarse tanto en el plano teórico como en el práctico.

Pese a todo lo anterior, aun cuando las redes satelitales ya consentían cierto tipo de retroalimentación en la comunicación, la educación a distancia adolecía de aquello que caracterizaba a la educación tradicional: la interactividad. Esta posibilidad de interacción, que desde siempre poseían los sistemas tradicionales, y sobre la cual se establecían precisamente las relaciones de poder que los caracterizan, era el último valladar antes de la aceptación completa de la educación a distancia como un modelo plenamente integral. Los cimientos para romper esta última barrera los estableció Berners Lee (Ortega, 2002), quien a finales de la década de los ochentas propuso un sistema de comunicación sumamente novedoso el cual permitía el manejo de medios diversos por una vía integrada, esto es, no sólo era multimediado, sino que además tenía un carácter mundial: el World Wide Web.

La implementación y el crecimiento exponencial de la Internet ayudaron a la educación a distancia a pasar a una cuarta etapa de desarrollo. Las posibilidades para la comunicación bidireccional que, tanto en el ámbito escrito como verbal o visual, permite este medio son casi infinitas pues admite la libre transferencia de datos en audio, video, gráficos y demás, lo que inmediatamente lo volvió el canal adecuadoparauncrecimientodesmedido de sus aplicaciones para la educación. La Internet, le permitió formalizar su presencia mediante el establecimiento de las llamadas universidades virtuales (Torres, 2001), cuyo antecedente se ubica en las universidades corporativas. La universidad virtual se basa en la utilización de la Web para generar ambientes virtuales de aprendizaje.

Los ambientes virtuales de aprendizaje son característicos de la actual fase evolutiva de la educación a distancia. 
Se encuentran basados en plataformas tecnológicas que utilizan a la Internet como herramienta de comunicación y soporte. Como un derivado de lo antepuesto, se han acuñado una serie de neologismos tales como; e-learning, tele enseñanza, tele educación, tele aprendizaje, campus virtual, educación vía Internet, universidad virtual o aprendizaje distribuido por Internet, entre otros. Nos parece significativo clarificar, luego entonces, el significado de esta variedad de conceptos pues el lector que se encuentre fuera del área de conocimiento puede interpretar que cada uno de ellos describe fenómenos diferentes. Tal y como lo plantea Belmonte Medina (2002), esta multiplicidad conceptual existente describe a un solo fenómeno: la educación virtual. Tal virtualidad, le permitiría a la educación a distancia iniciar la migración a una quinta etapa de desarrollo, misma que abordaremos en el último apartado del presente trabajo.

\section{La educación a distancia en la actualidad: avances $\mathbf{y}$ retrocesos}

La utilización de la red Internet permite a la educación a distancia desarrollar una serie de acciones que, mediante otros medios de comunicación, anteriormente, era imposible. Este medio es integrado, flexible y sumativo, pues dentro de su operación coexisten una serie de herramientas y opciones factibles de ser utilizadas con fines educativos. Es flexible porque permite desarrollar e integrar múltiples $\mathrm{y}$ variados procesos, herramientas $\mathrm{y}$ medios sin perder su operatividad y carácter original.
Es sumativo dado que su operación es escalable permitiendo integrar, regenerar $y$ enriquecer medios $y$ herramientas previamente constituidas, dando lugar a un poderoso medio de comunicación multimediado. Mediante la Internet, la educación a distancia en su fase virtual puede, en la actualidad, desarrollar lo que en otras etapas evolutivas de este modelo educativo era imposible: una escuela en la red.

Esa escuela virtual, aula virtual o campus virtual, resulta ser un sitio dotado de una interface gráfica y soportado por una compleja base de datos (una plataforma) que permite, mediante su acceso y manipulación, desarrollar todos los procesos y acciones que se efectúan en una escuela físicamente existente. Esta escuela virtual accede a desarrollar no solamente el proceso educativo en su nivel pedagógico y académico, también permite el tratamiento de los procesos administrativos que lo acompañan, así como el ambiente de comunicación y convivencia entre los actores que se da en una escuela presencial. Para lo antepuesto, es indispensable contar con una plataforma tecnológica o Learning Management System (LMS) por su definición inglesa, esto es, la operación de un software arquitectónicamente avanzado que permita desarrollar todas las funciones programadas para una escuela. Esta plataforma ha sido definida como un ambiente educativo de aprendizaje distribuido, sincrónico y asincrónico que permite interactuar remotamente a través de un conjunto de bases de datos. 
En mayor o menor medida en la educación a distancia desarrollada mediante la Web, de acuerdo con Duart y Sangrá (2002), se basa en por lo menos tres modelos distintos de operación: a) Los modelos centrados en los medios, b) Los modelos centrados en el profesor y c) Los modelos centrados en el alumno.

Los modelos centrados en los medios tienen la característica de que su foco de atención se centra en la herramienta tecnológica que se utiliza para la distribución de sus cursos, restándole importancia a los dos máximos protagonistas del acto formativo: el profesor y el estudiante. Este modelo, se ha vuelto extremadamente común en la actualidad. Muchas personas e instituciones, en la práctica, han dado por hecho que la simple utilización de las nuevas tecnologías, así como sus particulares herramientas pueden sustituir en mayor o menor medida no solo a los otros dos actores del evento, sino también a los distintos procesos entrelazados.

Por su parte, los modelos centrados en el profesor reproducen los esquemas operativos clásicos de un sistema educativo tradicional, es decir, se centra en la enseñanza y el profesor continúa siendo el foco de atención $\mathrm{y}$ único poseedor y transmisor del conocimiento, pero utilizando las nuevas tecnologías para ampliar el alcance de sus clases. Finalmente, los modelos centrados en el estudiante o en el aprendizaje son aquellos que tienen como eje al estudiante y sus procesos cognitivos. Sin embargo, este modelo que debiese ser el adecuado, no es precisamente el más común, pues dichos modelos, se han manifestado más en términos declarativos que en lo operativo.

Dado que la tendencia mundial se orienta precisamente a centrar todo el proceso de aprendizaje del estudiante, la mayoría de las instituciones se declaran seguidoras de dicho modelo, pero en la práctica se sigue operando de una manera tradicional. De hecho, “ [...] los modelos actualmente más centrados en el estudiante son los que se basan, fundamentalmente, en el autoaprendizaje o la autoformación" (Duart y Sangrá, 2002, p. 26).

La comprensión de estos modelos permitirá abordar algunas de las diferencias cualitativas que se presentan, en la concepción y operación de los ambientes virtuales de aprendizaje, independientemente del término con que se les denomine. El problema que reflejan estos tres modelos es una falsa concepción de la educación a distancia y concretamente en aquella que se basa en ambientes virtuales de aprendizaje.

Es importante notar que puede diseñarse y operar un ambiente de aprendizaje tanto para una escuela tradicional como para una escuela o campus en la red. Lo anterior significa que se debe de considerar claramente el enfoque didáctico y pedagógico, la tecnología, la administración del modelo, así como los actores. Ello implica entender que son paradigmas diferentes, mas no opuestos, y que en cierta medida pueden ser complementarios, -pero primero habrá de tenerse en cuenta que es más fácil extrapolar lo que ocurre en un modelo virtual a uno presencial que a la inversa-. En este sentido, el ambiente de aprendizaje resulta de la interacción de los sistemas: tecnológico, académico- 
pedagógico y el sistema administrativo. Estos tres sistemas interactúan de una manera secuencial y consecutiva, concretándose en el sitio y su respectiva interfaz gráfica existente en la Internet (García, 2005b, 2005c, 2006d).

En la actualidad, la escuela tradicional intenta retomar dentro de sus limitantes institucionales esta diferencia cualitativa, sin embargo, en la mayoría de los casos se han trasladado los vicios e inoperancias de la educación presencial tradicional a la educación virtual.

\section{Currículo y mundos virtuales: un breve acercamiento al futuro del aprendizaje}

Independientemente de los límites propios de la infraestructura comunicacional que soporta a la Internet, como ya explicamos, se han ampliado los canales de comunicación que permiten la interactividad de los actores del hecho educativo, tanto o más de lo que concede la educación presencial. Superada esta barrera que, conforme se robustecen las nuevas tecnologías de la comunicación y la información (NTIC), será eliminada progresivamente.

Queda pendiente otra limitante que tiene que ver con aspectos de orden físico, concretamente de infraestructura educativa, así como de orden didáctico-pedagógico, nos referimos al aprendizaje de habilidades y destrezas especializadas, así como la puesta en práctica delos conocimientos adquiridos en el nivel de experimentación o demostración.
En tal sentido, en la educación tradicional los estudiantes pueden acceder a los laboratorios y talleres tanto para la adquisición de habilidades y destrezas nuevas o especializadas, así como para la comprobación o corroboración de los conocimientos o principios aprendidos en un nivel teórico. Esto es, hasta el momento, relativamente imposible para un modelo educativo a distancia en su fase virtual.

Si bien existen ya una gran cantidad de materiales demostrativos tales como los tutoriales, software especializado y ciertos programas de simulación, todavía no se logran los grados de complejidad que permite un laboratorio o un taller. La educación a distancia se encuentra en los límites para ser una opción verdaderamente sustantiva, realmente innovadora y, consideramos, en muchos casos posiblemente mejor que la educación presencial tradicional. Tal afirmación se finca en la gestación, de una quinta etapa en su desarrollo que puede ser fundamentada en distintas iniciativas tecnológicas como las siguientes:

- La realidad virtual (RV)

- De inmersión total

- De inmersión parcial o de "escritorio"

- La realidad aumentada

- Los potenciales usos -a mediano plazo-, de las nanotecnologías

No todas estas opciones técnicas se encuentran ya en uso para efectos educativos, de hecho, en el caso de las nanotecnologías aún se ubican en una fase de desarrollo y exploración. 
Pese a lo anterior, y como preámbulo antes de abordar cada una de ellas, nos gustaría retomar un problema reiteradamente genérico cuando se utilizan las nuevas tecnologías de la información y la comunicación (NTIC) en la educación: el problema curricular.

En consideración a lo antepuesto, sería un gran engaño pensar que el simple acceso al hardware (tal como equipos de cómputo con la capacidad suficiente para su uso en contenidos que implican alta resolución, gafas estereoscópicas, guantes sensitivos, etc.) que nos permita iniciarnos en el uso de la RV o la RA como un apoyo tecnológico para fines educativos es suficiente para implementar un cambio.

Pensar que con ello es suficiente, estaríamos cayendo -una vez más-, en un terrible error. Para su utilización -dentro del ámbito educativo-, se necesita recorrer aún un gran trayecto que pasa no solamente por el ajuste de las políticas educativas sino también del currículo escolar. En lo relativo a la política educativa, se debe considerar que las verdaderas potencialidades de las nuevas tecnologías se encuentran mucho más lejos que el simple hecho de la distribución de; tablets o laptops, $y$ que el real problema se ubica en su uso educativo por lo que, el aprendiz $y$ el docente, no arriban de manera inmediata al futuro por la única posesión de dichos aparatos.

Nos parece que se deben tomar decisiones para establecer -en el lugar que se considere pertinente-, laboratorios de desarrollo de software educativo donde se consideren las tecnologías antes enlistadas. Esto mismo, por otro lado, puede acontecer para efectos instructivos menos formales tales como la capacitación o la educación continua, dirigida al sector profesional o laboral. Por lo que respecta a la instrucción formal, es precisamente en este punto donde se ubica el ajuste curricular puesto que se pueden rediseñar asignaturas o módulos, -dependiendo del enfoque de cada plan o programa educativo-, completamente basados en las potencialidades de las tecnologías RV o RA, los resultados de ambas condiciones, es propio afirmar, serían factibles de ser aplicados tanto en la educación presencial como en la virtual.

A manera de paréntesis, es muy significativo tener presente siempre que -en general-, cuando las nuevas tecnologías se dirigen al ámbito educativo, su manipulación y diseño de los trabajos resultantes susceptibles de utilizar tanto de manera presencial en el aula como en modelos de aprendizaje virtual, deben partir de un ingenioso trabajo interdisciplinar ya que por lo que hemos visto, hasta el momento, se ha caído en un diálogo de sordos donde los diseñadores -generalmente ingenieros que conocen de programación y computación-, son quienes terminan produciendo programas que en muchos casos carecen de una estructura pedagógica y acaban siendo herramientas muy burdas.

La falta de pericia educativa o de diseño pedagógico termina por repetir los mismos vicios de la escuela tradicional pero ahora basada en modelos y herramientas con características -tecnológicamente avanzadas-. Esto, en términos cualitativos, sigue siendo 
la repetición de métodos de enseñanza arcaicos y por ende poco susceptibles de aprendizaje. Dentro de los aspectos curriculares a ser tomados seriamente en cuenta, se ubican la utilización de una serie de técnicas de aprendizaje basado en lo siguiente: a) el juego, b) los errores, c) los problemas, d) las historias, e) los equipos de trabajo, f) el aprender haciendo y g) aprendizaje por proyectos, etc.

Habrá que considerar firmemente, por otro lado, que los seres humanos aprendemos básicamente haciendo y manipulando cosas y que el aprendizaje puramente descriptivo o conceptual, es lo más fácil y rápidamente olvidable, tal como se presenta en la siguiente figura.

\section{Ilustración 1. Cono de aprendizaje según Edgar Dale}

\begin{tabular}{|c|c|c|}
\hline $\begin{array}{l}\text { Despues de } 2 \text { semanas } \\
\text { tendemos a recordar }\end{array}$ & \multicolumn{2}{|c|}{$\begin{array}{l}\text { Naturaleza do la } \\
\text { actividad involucrada }\end{array}$} \\
\hline El $10 \%$ de lo que leemes & \multicolumn{2}{|r|}{ Actividad verbal } \\
\hline El $20 \%$ de lo que oimos & \multicolumn{2}{|c|}{ Palabras oidas } \\
\hline El $30 \%$ de lo que yemos & \multicolumn{2}{|c|}{ Dibujos observados } \\
\hline $\begin{array}{l}\text { El } 50 \% \text { de lo que } \\
\text { eimos y vemos }\end{array}$ & \multicolumn{2}{|r|}{ Actividad visual ไృ } \\
\hline $\begin{array}{l}\text { El } 70 \% \text { de lo que } \\
\text { decimos }\end{array}$ & $\begin{array}{l}\text { bate } \\
\text { ción }\end{array}$ & $\begin{array}{l}\text { Actividad } \\
\text { participativa y } \\
\text { receptiva }\end{array}$ \\
\hline $\begin{array}{l}\text { El } 90 \% \text { de lo } \\
\text { que decimos } \\
\text { yhacemos }\end{array}$ & $\begin{array}{l}\text { Ón teatral } \\
\text { reales } \\
\text { a aprender }\end{array}$ & $\begin{array}{l}\text { Actividad } \\
\text { pura }\end{array}$ \\
\hline
\end{tabular}

Fuente: Dale, Edgar en Landolfi, Hugo. (2018). Cursos de Liderazgo a distancia, consultado en noviembre de 2018 en http://www.sabiduria.com/

$\underline{\text { liderazgo/crisis-educativa/ }}$

En consideración a tal esquema, es que el aprendizaje básicamente ocurre en un plano en el que concurre lo que decimos y hacemos. De esta forma, una de las experiencias que vale la pena retomar, para fines educativos, es aquella derivada de la industria del entretenimiento. La mayoría de sus productos (todavía cercanos a la realidad virtual no inmersiva o de "escritorio") tal como lo plantea De Antonio, Villalobos \& Luna (2000), es lo que más ha permeado en las diferentes capas poblacionales completamente integradas a la cultura digital, luego entonces, su experiencia puede ser derivada al ámbito educativo. Ello, además, sería una magnífica oportunidad para reconsiderar al juego o los ambientes lúdicos como una acción que deriva en un alto potencial de aprendizaje a todas edades y niveles educativos. El juego como bien afirma Montero, Dávila y Tejero (2010) y Schank (1997), posee intrínsecamente las mismas características del aprendizaje en primera persona, como se profundizará un poco más adelante. A continuación, procuraremos abordar, de manera sucinta, cada una de las tecnologías susceptibles de ser utilizadas para fines educativos y enlistados con anterioridad.

El primer caso del que nos ocuparemos es el de la Realidad Virtual (RV), misma que puede ser definida como "[...] un medio tecnológico compuesto por una simulación tridimensional por computadora, donde el participante utiliza varios sentidos sensoriales para interactuar con dicha simulación, dando en ésta la sensación de estar mentalmente inmerso" (García, 2015, p. 2).

La RV -sobre todo la sustentada en la inmersión total-, impacta las áreas cognitiva, afectiva y sensitiva del aprendiz y se deriva de la utilización 
tanto de software como de hardware especializado, dirigido a la recreación de mundos virtuales tridimensionales, mismos que conforman un gran reto no sólo para las teorías del aprendizaje tradicionales, los métodos pedagógicos o la didáctica utilizada hasta el momento dentro de la educación.

Dicha tecnología innova los esquemas de aprendizaje ya que como bien afirma Vera, Ortega y Burgos (2003), privilegian el aprendizaje en primera persona, mismo que se presenta cuando el sujeto aprende de una manera natural, cotidiana y subjetiva lo que incrementa la comprensión, el recuerdo y la solución creativa de los problemas y sucesos del entorno. Tal condición se opone al aprendizaje en tercera persona que es utilizado en la escuela tradicional y que -lamentablemente hasta ahora-, también ha sido trasladado a la mayoría de los programas de educación virtual que se centran más en el maestro y la tecnología que en el aprendiz.

Los diseños operativos de la RV también marcan la pauta para nuevos enfoques investigativos, ya que tales mundos aun cuando no totalmente inmersivos modifican mediante los juegos de video -de hecho, ya lo están haciendo-, los sustratos cognitivos humanos, así como su sensibilidad y afectividad, además de cambiar sus esquemas de relaciones sociales con otros miembros de la comunidad. La RV de inmersión total -como muchos otros de los artefactos o cosas utilizadas actualmente en la vida cotidiana-, tiene parte de su origen en el ámbito militar, donde se comenzaron a desarrollar simuladores para la capacitación de pilotos. Otro de su uso se ubica en las consultorías para la capacitación de personal, aún cuando -se debe de reconocer -, no se ha hecho extensiva su utilización y que son -sobre todo-, las grandes compañías quienes la han venido implementando.

Las simulaciones en computadora son necesarias debido a que los entrenamientos por aprendizaje directo pueden ser poco prácticos y que aprender en el trabajo, de igual manera, sería potencialmente peligroso tanto para la empresa como para los trabajadores. "Para aprender, usted necesita un ambiente en el cual pueda aprender. El aprendizaje virtual se lleva a cabo en un ambiente virtual" (Schank, 1997).

Es importante considerar, por otra parte, que muchos de los programas y tendencias actuales de la RV, -sobre todo de inmersión parcial- se deben a la industria del ocio en los juegos de video o por computadora pero básicamente, la educación aún no ha sido totalmente impactada, aún cuando se hallan sitios tales como Second $\mathrm{Life}^{3}$, que son un excelente ejemplo de la RV de escritorio y que tiene ciertos aspectos y espacios educativos.

Creemos que existen varios problemas para la inserción de la RV de inmersión total dentro del ámbito educativo. En nuestro país (México), pese a existir algunos casos de acercamiento a tal problemática (Gonzáles y Chávez, 2011) y (García, 2015), los esfuerzos son

$3 \mathrm{http://secondlife.com/?lang=es \& utm} \mathrm{source=-}$ Google\&utm medium=textspan\&utm term=Spanish Search\&utm content=Brandspanish\&utm campaign=SecondLifeenEspanolChat\&gclid=CJ6I 6nK98gCFQIOaQodvacCSw. 
casi nulos por diversas circunstancias. Una de las más comunes es considerar que se pueden generar softwares, simuladores virtuales $\mathrm{u}$ objetos de aprendizaje puramente desde la visión de los desarrolladores sin tomar en cuenta a los especialistas tanto del ámbito educativo como disciplinar, tal como ya se planteó.

Por otro lado, y dentro del ámbito de la educación virtual, dado que las plataformas de distribución de educación a distancia se han vuelto cada vez más accesibles y amables en su uso, cualquiera puede "subir" cursos y contenidos sin pasar por algún tamiz verdaderamente didáctico y pedagógico con el consecuente resultado de la vulgarización educativa y el uso indiscriminado de las Learning Management System (LMS) como un simple pizarrón en la red. En este tenor de ideas, existen cursos que son únicamente la traslación de las viejas prácticas escolares a un ambiente Web, lo cual es inadmisible. Por poner un ejemplo, todas aquellas carreras o cursos cercanos al ámbito de la ingeniería que se ofertan en la red de Internet por las características propias de tales carreras, mismas que, por obligación para su enseñanza $y$ aprendizaje, necesitan prácticas fuertemente centradas en lo procedimental -que sólo pueden ser desarrolladas en laboratorios o talleres-, representan en la actualidad lo más cercano a un fraude educativo.

Como puede evidenciarse, el problema curricular es recurrente, en tal sentido, lo mínimo indispensable, en caso de no poder acceder físicamente a los talleres o laboratorios, es la utilización de simulaciones virtuales con capacidad de manipulación $\mathrm{y}$, por tanto, alta interactividad por parte del estudiante usuario. La otra opción, no excluyente con la anterior, es utilizar la RV no inmersiva o de escritorio ya que -hasta donde sabemos-, la RV de inmersión total no está en uso, cuando menos no en nuestro país. Aun así, el simple hecho de diseñar simuladores, programar su uso y dosificar los contenidos curriculares implica un trabajo interdisciplinario, mismo que no se puede obviar simplemente por la actual flexibilidad, en el caso de la educación virtual, de las plataformas para la enseñanza a distancia.

Otra circunstancia que obstaculiza el uso de la RV de inmersión total en la educación en general, es la poca accesibilidad del usuario de los equipos físicos necesarios por cuestiones económicas. Pese a lo anterior, las instancias educativas estatales públicas, pero también algunas privadas, pueden auspiciar su adquisición e iniciar el desarrollo de contenidos para su utilización. Esto muchas veces no ocurre pues una de las preocupaciones constantes, en términos administrativos, se ubica en la denominada "tasa de retorno", es decir, la posibilidad de recuperar a corto o mediano plazo la inversión efectuada. Creemos que a diferencia de los contenidos dirigidos -por lo menos-, a la educación virtual que actualmente pululan en la red (compuestos básicamente textos en formato PDF, algunos videos o películas, exposiciones en power piont, algunos objetos de aprendizaje, etc.), los contenidos prediseñados para ser utilizados con la RV son mil veces más 
estables y rentables a corto y mediano plazo con lo que la tasa de retorno sería positiva.

Para concluir la presente temática, podemos decir que si bien, hasta el momento, para el común denominador de la gente, y particularmente los docentes y los estudiantes, la RV es aún un ente extraño, su uso en situaciones de alto riesgo en la industria y la medicina es algo más que común. Por otro lado, la venta de los aparatos periféricos para su uso cotidiano se encuentra en curso y que, como todo lo que acontece en la actualidad con el ámbito tecnológico, su popularización se ha ido incrementando. El problema que se está presentando es que es la industria del entretenimiento quien la está acaparando y no la educación.

A continuación, abordaremos otra tecnología que puede apoyar fuertemente el aprendizaje $\mathrm{y}$, por lo tanto, también en suficiencia a la educación tanto presencial como virtual: la realidad aumentada.

Otro de los tópicos dentro de nuestro listado de opciones tecnológicas para la generación de mundos virtuales dirigidos a la educación, se encuentra representado por la RealidadAumentada (RA). Esta tecnología aún cuando su concepción no es completamente reciente (se conceptualizó en el inicio de la década de los 90 con algunos fines lúdicos), en la actualidad, es un evento que es posible ser llevado a la práctica cotidiana con ciertos grados tanto de simplicidad como de complejidad.

Se puede definir a la RA como "[...] una tecnología que ofrece una forma novedosa de interacción con el usuario, permitiendo la presentación de elementos reales y virtuales en un mismo ambiente" (Gil y otros, 2014, p: 941). Es decir, es una aplicación mixta que combina los planos de la realidad verdadera o física con una visión holográfica que aumenta la percepción de los sujetos sobre los objetos susceptibles de ser conocidos, tocados, explorados y por lo tanto aprendidos. A diferencia de la realidad virtual inmersiva, podemos decir que los aprendices mantienen un pie en la realidad física y el otro en la virtual. Básicamente nos encontramos en un modelo hibrido entre la virtualidad completa y la normal, en tal sentido $\mathrm{y}$ siguiendo a Flores Cueto y otros (2010), una similitud entre ambas realidades podría estar representada -de manera metafórica-, por los libros infantiles que siguiendo un modelo de ambiente plano y secuencial para su lectura y presentación, internamente guardan páginas u hojas completas que al abrirlas exhiben esquemas a escala que cambian de dimensión y por lo tanto, la percepción y el aprendizaje de quien lo utiliza, tal y como se presenta en la siguiente imagen.

\section{Ilustración 2. Libro con representaciones pictóricas tridimensionales a escala}

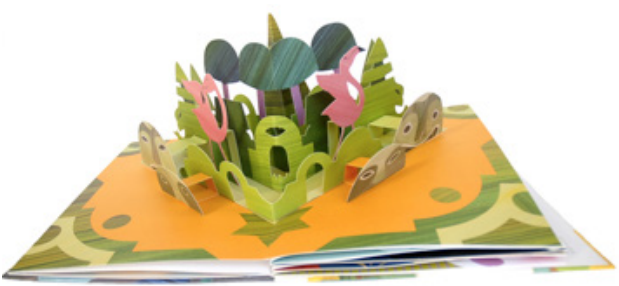

Fuente: Torrubia, Isabelle. (2013). Agencia Literaria, consulta realizada en marzo de 2014 n http://www.torrubia-agency.com/ alizarine/2013/01/16/libro-taller-pop-up/ 
La RA ofrece muchas posibilidades para su uso educativo, resulta ser muy atractiva -por ejemplo-, para los recorridos en museos, la enseñanza de la anatomía, el manejo de máquinas y conceptos complejos en un ambiente real donde el estudiante o sujeto interactuante puede literalmente "meter las manos" ante el objeto que se encuentra en frente, algunos excelentes ejemplos se pueden ubicar en dos video de Youtube: VISA Augmented Reality Campaign ${ }^{4}$, Live Augmented RealityNational Geographic ${ }^{5}$

Esta circunstancia es significativa pues puede ser usada tanto para una clase en aula tradicional como en un ambiente virtual basado en una LMS. En tal sentido, Flores Cueto y otros (2010) nos indica que "[...] cuando tomamos en cuenta la realidad aumentada como herramienta para dar creatividad a nuevas metodologías pedagógicas, permitimos al estudiante experimentar la interacción con objetos virtuales que refuercen las clases presénciales, material impreso de apoyo entre otros modelos pedagógicos tradicionales" ( $\mathrm{p}$. 7). A pesar de lo anterior, la RA ha sido poco utilizada en proyectos educativos, por lo que resulta importante su investigación y uso.

Como último tema del presente capítulo abordaremos -muy concisamente-, las potencialidades de la nanotecnología en la ampliación de los horizontes del 4 Youtube, consulta realizada el 16 de mayo de 2019 en: https://www.youtube.com/ watch?v=HqMVZmOkIW0

5 Youtube, consulta realizada el 16 de mayo de 2019 en: https://www.youtube.com/ watch?v=D0ojxzS1fCw aprendizaje, como acción y elemento potenciador tanto de la RV como de la RA. Las nanotecnologías, siguiendo el discurso de Miguel Aznar (2013): “[...] es la comprensión y el control de la materia en dimensiones de aproximadamente 1 a 100 nanómetros, donde fenómenos únicos permiten nuevas aplicaciones" (p. 5).

Elementalmente, cuando hablamos de esta ciencia interdisciplinaria, nos referimos al mundo de lo microscópico, de las relaciones entre partículas difícilmente factibles de ser observadas -y manipuladas-, a simple vista por el ser humano. Es el mundo de los sustratos mínimos que, para acceder a ellos, se necesitan aparatos complejos tanto para su conocimiento como su manejo.

Además de lo antepuesto, la nanotecnología permite al ser humano la fabricación de compuestos moleculares o atómicos que pueden tener características distintivas como la autorreplicación y reproducción masiva, aparte de ser transmisores de información o bien que -mediante su recombinación-, se generen materiales especiales no necesariamente existen en el ambiente natural del ser humano. La nanotecnología, como un instrumento aplicable a la educación, aún se ubica un poco lejos. Lo que se puede especular es que su avance serviría en dos niveles distintos: a) La miniaturización $\mathrm{y}$, por lo tanto, potenciación de las computadoras y los dispositivos móviles, y b) El diseño de micro implantes que "bombardeen" zonas cerebrales para el aprendizaje de ciertas rutinas, conocimientos o -dentro 
del ámbito lúdico-, la estimulación de cinturones de placer.

Hablamos, básicamente de planteamientos teóricos con cierta calidad especulativa, aún falta saber si ello es o será posible. En todo caso, nosotros consideramos que al igual que la mayoría de las ciencias relativas al siglo XXI (biotecnología, geomática, ciencias cognitivas, etc.) tales eventos serán posibles con el tiempo.

\section{Conclusiones}

El desarrollo de la realidad virtual de inmersión total y -en su momento las nanotecnologías-, con fines educativos, permitirán superar las barreras existentes para la educación a distancia, relacionadas con aquellas prácticas $\mathrm{y}$ aprendizajes que implican un alto contenido procedimental -prácticas en laboratorio, taller, quirófano, etc.-, que pueden implicar no sólo un alto riesgo humano sino también económico. La RV y la RA aplicadas a la educación virtual permitirán -ahora si- aprender en todo momento, lugar y circunstancia.

La RV y la RA ampliarán -inclusive-, las potencialidades del aprendizaje, dado que en su práctica y aplicación se implicará todo el sistema perceptual y cognitivo de los aprendices, cosa que no ocurre en una situación real. Su uso y aplicación en la educación, -desgraciadamente-, también ampliará la distancia existente entre las franjas poblacionales que ya están encaminadas en la cultura digital y acelerará, por lo tanto, la generación de nuevos analfabetas digitales y marginados sociales.
También aumentará el riesgo de la pérdida del libre albedrío en muchos sentidos, toda vez que se generan posibilidades tales como los mensajes de carácter subliminal que entrarían de manera directa a la conciencia del aprendiz. Para finalizar, podemos sostener que, en la actualidad, la educación a distancia se encuentra ubicada en la etapa virtual. Este modelo educativo ha trascendido, con base en el desarrollo de las Tecnologías de la Información y Comunicación (TIC), las barreras y límites impuestos por un mundo analógico para desenvolverse en un universo digital que se vería ampliado enormemente con el uso de la RA y la RV de inmersión total.

\section{Referencias bibliográficas}

Aznar, Miguel. (2013). Pensamiento crítico acerca de la nanotecnología. Revista Digital Universitaria (14) 4. Recuperado de: http:// www.revista.unam.mx/vol.14/num4/art35/ art35.pdf

Belmonte, Medina Andrés. (2002). Estudios de los Sistemas Telemáticos de Formación basados en Internet. Recuperado de: http://www. telematicotransportes-ftcm.org/contenidos/ contenidos.html

DeAntonio,A., Villalobos, M. \& Luna, E.(2000). Cuándo y cómo usar la realidad virtual en la enseñanza. Revista de Enseñanza y Tecnología (26), 26-36. Recuperado de: documat.unirioja. es/descarga/articulo/4794517.pdf

Duart, J. \& Sangrá, A. (2002). Aprender de la virtualidad. España: Gedisa. 
Educación a Distancia. (s.f.). Educación a Distancia. Recuperado el 23 de septiembre de 2002, del sitio Web de la organización EDUCADIS, https://www.educadis.com/

Flores, Cueto Juan José y otros. (2010). La realidad aumentada como herramienta para mejorar los procesos educativos en la USMP. Revista Enlace Virtual. No. 10. Recuperado el 10 de mayo de 2019, del sitiohttp://www. ibertic.org/evaluacion/sites/default/files/ biblioteca/14_realidad aumentada.pdf

García Ruiz, Miguel Ángel. (2015). La tecnología de la realidad virtual aplicada a la educación. Recuperado de: www.hrl.uoit. ca/ miguelga/Realidad_virtual_educacion Iridia.pdf

García, J. (2005a). Panorama evolutivo de la educación a distancia. Revista Contexto educativo, (36). Recuperado de: http://www. contexto-educativo.com.ar/2005/3/nota-08.htm

García, J. \& Castillo, A. (2005b). Los componentes de un sistema de educación virtual: el sistema académico-pedagógico. Revista electrónica de pedagogía Odiseo, (4). Recuperado de: http://www.odiseo.com. $\underline{\mathrm{mx} / 2005 / 01 / \text { index.htm }}$

García, J. \& Castillo, A. (2005c). Los componentes de un sistema de educación virtual: el sistema tecnológico. Revista electrónica de pedagogía Odiseo (5). Recuperado de: http:// www.odiseo.com.mx/2005/02/index.htm

García, J. (2006d octubre). La configuración organizacional de los sistemas de educación virtual: una asignatura pendiente. Facultad de Ciencias Humanas Unidad Mexicali de la Universidad Autónoma de Baja California (UABC), Baja California, México.

Gil, Gustavo y otros. (2014). Implementación de Objetos de Aprendizaje Con Realidad Aumentada en la Educación. Trabajo presentado en el Seminario WICC 2014 XVI Workshop, Salta, Argentina.

González, A. \& Chávez, G. (2011). La realidad virtual inmersiva en ambientes inteligentes de aprendizaje. Revista Icono (14) 2. Recuperado de: $\quad$ http://www.icono14.net/ojs/index.php/ icono14/article/view/42

Montero, E., Dávila, M. \& Tejero, B. (2010). Aprendiendo con videojuegos: jugar es pensar dos veces. España: Narcea.

Ortega, J. (2002). Planificación de ambientes de aprendizaje interactivos on-line: Las aulas virtuales como espacios para la organización y el desarrollo del teletrabajo educativo. Universidad de Granada, Granada, España. Recuperado de: http://www.tecnologiaedu. us.es/gid-fete/paginas/p3.htm

Schank, Roger. (1997). Aprendizaje virtual. McGraw/Hill: México.

Torres, A. (2001). La Universidad Virtual: Educación Para la Sociedad del Conocimiento. México: Delfos.

Vera O., Ortega, A. \& Burgos, Á. (2003). La realidad virtual y sus posibilidades didácticas. Revista Etícanet (2). Recuperado de: www. ugr.es/ sevimeco/revistaeticanet/Numero2/.../ Realidadvirtual.pdf 